\title{
Asymmetric Dominance, Deferral and Status Quo Bias in a Behavioral Model of Choice*
}

\author{
Georgios Gerasimou ${ }^{\dagger}$
}

April 9, 2015

\begin{abstract}
This paper proposes and axiomatically characterizes a model of choice that builds on the criterion of partial dominance and allows for two types of avoidant behavior: choice deferral and status quo bias. These phenomena are explained in a unified way that allows for a clear theoretical distinction between them to be made. The model also explains the strengthening of the attraction effect that has been observed when deferral is permissible. Unlike other models of status-quo biased behavior, the one analysed in this paper builds on a unique, referenceindependent preference relation that is acyclic and generally incomplete. When this relation is complete, the model reduces to rational choice.
\end{abstract}

Keywords: Partial dominance; incomplete preferences; choice deferral; status quo bias; attraction effect

JEL Classification: D01, D11

${ }^{*}$ I thank Mark Dean, Robert Evans, Yusufcan Masatlioglu, Paola Manzini, Marco Mariotti, John Quah, Ran Spiegler, Jörg Stoye, audiences at Cambridge, Amsterdam, Queen Mary-London, St Andrews, EBIM 2010 (Bielefeld), EEA-ESEM 2011 (Oslo), SIRE-BIC 2012 (Edinburgh), and especially two referees of this journal for their very useful comments. Some of the material presented in this paper first appeared in preliminary form in working papers titled "Rational indecisive choice" (2010) and "Asymmetric dominance, deferral and status quo bias in a model of choice with incomplete preferences" (2012). Partial financial assistance from the Scottish Institute for Research in Economics is gratefully acknowledged. Any errors are my own.

${ }^{\dagger}$ School of Economics \& Finance, University of St Andrews. Email address: gg26@st-andrews.ac.uk. 


\section{Introduction}

Decision problems may be of two types, depending on the nature of their status quo. The first type includes problems where the status quo enters as an option that is qualitatively similar to all others. A consumer would be facing a decision problem of this type if he was endowed with insurance policy $A$ and was confronted with the choice between keeping $A$ or giving it up for one of policies $B$ or $C$. The second type includes decision problems where the status quo is not of the same nature as the other feasible alternatives. In a modification of the previous example, the consumer would be facing a problem of this type if he was uninsured and policies $A, B$ and $C$ were available to him. Maintaining the status quo in problems of the former type translates into choosing a physical alternative. By contrast, status quo maintenance in problems of the latter type translates into choosing no physical alternative. In this paper, the former type of problems will be referred to as being associated with a specific status quo and the latter as ones with a non-specific status quo.

Choice deferral refers to the observation that, when faced with decision problems with a non-specific status quo, people sometimes choose none of the options available to them, or equivalently choose their non-specific status quo. Some reasons for such behaviour include undesirability of the feasible alternatives, difficulties in making comparisons and finding a dominant option that are caused by decision conflict, and choice overload (Zakay, 1984; Tversky and Shafir, 1992; Greenleaf and Lehmann, 1995; Dhar, 1997; Luce, 1998; Iyengar and Lepper, 2000; Dhar and Simonson, 2003). Importantly, when decision conflict is the reason why the agent defers, it is understood that he does so despite the fact that all alternatives are desirable in the sense that each of them would be chosen had it been the only feasible option. On the other hand, status quo bias or default bias refers to the observation that, when faced with decision problems with a specific status quo, individuals more commonly choose a feasible option when this is actually their status quo than when it is not (Knetsch and Sinden, 1984; Knetsch, 1989; Samuelson and Zeckhauser, 1988). Behaviour that exhibits choice deferral or status quo bias is sometimes called avoidant behaviour (Anderson, 2003).

This paper uses the expanded choice domain introduced in Masatlioglu and Ok (2005) and extends the model of partially dominant choice proposed in Gerasimou (2015) in the direction of allowing for both types of avoidant behavior. This model is the first in the literature to feature a decision maker whose choices are guided by an acyclic and incomplete preference relation that is independent of the decision problem's type and menu of feasible alternatives. The way this preference relation is used by the agent is similar across the two types of decision problems. Specifically, in problems with a non-specific status quo, the decision maker chooses an option if and only if it dominates (i.e. is preferred to) some feasible alternative and is not dominated by any such alternative. The agent defers choice when and only when such a feasible option cannot be found. In problems with a specific status quo, on the other hand, he 
chooses an option other than the status quo if and only if the option is undominated and dominates the status quo, while the latter is chosen if the above is not the case for any alternative.

The model explains choice deferral that is due to incomparability/decision conflict. Moreover, in contrast to existing models, it also formalizes directly, by means of a single preference relation, the explanation of status quo bias that is based on preference incompleteness (Bewley, 2002; Mandler, 2004; Masatlioglu and Ok, 2005). As such, it relies on neither the use of multi-functions (Masatlioglu and Ok, 2005) nor on multiple, reference-dependent preference relations (Tversky and Kahneman, 1991; Bleichrodt, 2007; Apesteguia and Ballester, 2009). In addition, the partial dominance structure of the model allows for a simple explanation of findings reported in Dhar and Simonson (2003) suggesting a strengthening of the attraction effect (Huber, Payne, and Puto, 1982) and a weakening of the compromise effect (Simonson, 1989) when deferral is permissible. Finally, this model appears to be the first in the literature to make a clear theoretical distinction between choice deferral and status quo bias, and also to suggest a theoretical connection between the potential drivers of the attraction effect and of status quo bias or avoidant behavior more generally.

The next section introduces the model, which is referred to as the extended partial dominance procedure. The section following it introduces and discusses the axioms that characterize this choice procedure and identifies the way in which utility maximization is included as a special case by the model whenever preferences are complete. Section 4 discusses the model's predictions in relation to the experimental evidence, provides a review of the related literature and concludes with some methodological remarks about two approaches in the modelling of choice deferral.

\section{Extended Partial Dominance}

Let $X$ be a finite set of alternatives and $\mathcal{M}$ the collection of all subsets of $X$. Elements of $\mathcal{M}$ are menus. Almost exactly as in Masatlioglu and Ok (2005), the set of decision problems $\mathcal{S} \subset \mathcal{M} \times \mathcal{M}$ is defined as the set of all pairs $(A, B)$, where $A \in \mathcal{M}, A \neq \emptyset$, and either $B=\emptyset$ or $B=\{s\}$ for some $s \in A$. Thus, $\mathcal{S}$ is divided into pairs of the type $(A, \emptyset)$ and $(A,\{s\})$, where $s \in A$. To be consistent with the notation in Masatlioglu and Ok $(2005),(A, \diamond)$ will be written instead of $(A, \emptyset)$, and $(A, s)$ instead of $(A,\{s\}) .{ }^{1}$ Consistent with the terminology that was introduced above, a pair $(A, \diamond)$ will be thought of as a decision problem with a non-specific status quo, and a pair $(A, s)$ is a decision problem with a specific status quo. ${ }^{2}$ A generic decision problem (i.e. with or without a specific status quo) will be denoted by $(A, p)$.

A choice correspondence is a mapping $C: \mathcal{S} \rightarrow X$ that satisfies $C(A, p) \subseteq A$ for all

\footnotetext{
${ }^{1}$ The object $\diamond$ is defined as something not belonging to $X$ in Masatlioglu and Ok (2005). This minor difference in the two definitions is unimportant mathematically.

${ }^{2}$ Other interpretations have been adopted in the literature. For instance, Masatlioglu and Ok (2015) call $(A, \diamond)$ as a problem without a status quo and $(A, s)$ as a problem with a status quo.
} 
$(A, p) \in \mathcal{S}$. Imposing nonempty-valuedness of $C$ for all decision problems of the second type is obviously necessary for ensuring conceptual relevance and internal consistency. Therefore, it will be assumed that, for all $(A, s) \in \mathcal{S}$,

$$
C(A, s) \neq \emptyset
$$

However, in order to capture the phenomenon of choice deferral, $C$ will generally be allowed to be empty-valued in problems with an irrelevant status quo. That is, there may exist $(A, \diamond) \in \mathcal{S}$ such that

$$
C(A, \diamond)=\emptyset \text {. }
$$

General nonempty-valuedness is introduced as an explicit axiom later and will be used to demonstrate how the model developed below includes rational choice as a special case. This approach to modelling deferral is discussed in section 4.3.

The behavioral model of choice that is proposed and analyzed in this paper is introduced next.

\section{Definition 1}

A choice correspondence $C: \mathcal{S} \rightarrow X$ is generated by an extended partial dominance $(E P D)$ procedure if there exists a unique acyclic relation $\succ$ on $X$ such that, for all $(A, \diamond),(A, s) \in \mathcal{S}$,

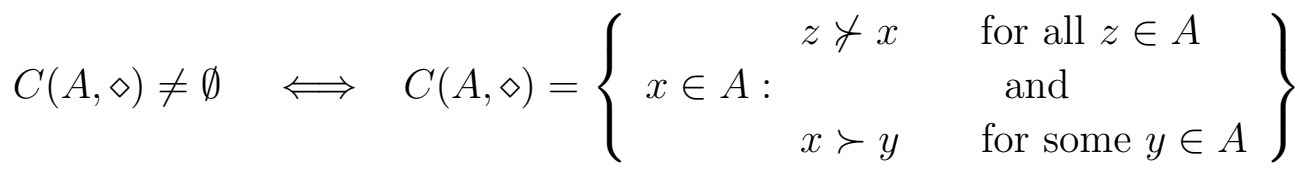

$$
\begin{aligned}
& C(A, s) \neq s \Longleftrightarrow C(A, s)=\left\{\begin{array}{ll}
x \in A: & \text { for all } z \in A \\
x \succ s & \text { and }
\end{array}\right\}
\end{aligned}
$$

The former part of the EPD model, (1a), implies ${ }^{3}$ that, for all $(A, \diamond) \in \mathcal{S}$,

$$
C(A, \diamond)=\emptyset \Longleftrightarrow x \nsucc y \text { and } y \nsucc x \text { for all } x, y \in A \text {. }
$$

Therefore, (1a) and (2) jointly dictate behavior in decision problems with a non-specific status quo. In particular, under the EPD model the agent defers choice at those and only those menus in which he is unable to make a preference comparison between any two alternatives, as captured in (2). When such a comparison can be made, however, the agent chooses among those feasible alternatives that are not dominated by anything in the menu and also dominate something, as captured in (1a). The difference between this part of the EPD model and the model of partially dominant choice proposed in Gerasimou (2015) is that, instead of considering everything to be

\footnotetext{
${ }^{3}$ This is so because the relation $\succ$ is acyclic. Thus, if (1a) is not satisfied in $(A, \diamond)$, it must be because $x \nsucc y$ for all $x, y \in A$.
} 
choosable when faced with a decision problem with a non-specific status quo in which no preference comparison can be made, the agent here always defers choice from such a menu.

Moreover, the second part of this model, (1b) implies that for all $(A, s) \in \mathcal{S}$

$$
C(A, s)=s \Longleftrightarrow z \nsucc s \text { for all } z \in A \text {. }
$$

Thus, (1b) and (3) jointly characterize behavior in problems with a specific status quo. This part of the model suggests that, in decision problems of this kind, the agent chooses something other than the status quo if and only if he can find an undominated feasible option that is preferred to it.

The link between (1a) and (1b) in the EPD model is noteworthy as it suggests that the agent's decision to abandon the status quo, as captured by (1b), follows a special application of the partial dominance choice procedure that is described in (1a). In particular, for a feasible alternative other than the status quo to be choosable it must be universally undominated on the one hand and partially dominant on the other, but the latter must happen not with respect to some arbitrary feasible alternative, but in relation to the status quo option instead. Thus, the model features a referencedependent, special application of the partial dominance choice procedure when it comes to the decision of giving up the status quo, and reveals a surprising connection between the potential drivers of the two phenomena. The descriptive relevance and limitations of the model are discussed in section 4.1 below.

Through the discussion of the experimental findings with which it is compatible it will become clear that the EPD model is one that predicts context-dependent choices. Importantly, however, it is not a model of context-dependent preferences. The reader is referred to Kriesler and Nitzan (2008) for arguments and evidence suggesting that context-dependent choice procedures with context-independent preferences are empirically relevant.

\section{The Axioms}

This section presents the axioms that characterize the EPD model, and establishes a formal axiomatic link between this model and utility maximization.

\section{A1: Bounded Status Quo Bias (BSQB)}

For all $x, s \in X$, if $x \in C(A, s)$ and $x \neq s$, then $x \in C(A, \diamond)$; and if $C(\{x, s\}, \diamond)=x$, then $C(\{x, s\}, s)=x$.

According to the first part of A1, whenever the agent is faced with a decision problem with a specific status quo and considers an alternative other than the status quo to be choosable, he also chooses that alternative when faced with the same menu and 
the status quo is non-specific. Indeed, in view of the evidence for status quo bias, one might expect the presence of a status quo to make it more difficult for him to declare choosable some other feasible option. Once this is the case, however, one would also expect the choosability of such an option to be maintained (if not increased) in the absence of the potentially "gravitating" forces that a status quo option may exercise on him.

Before discussing the second part of the BSQB axiom it is worth noting that one natural interpretation of the statement $C(\{x, s\}, \diamond)=x$ is that the agent would almost always choose $x$ and reject $s$ when faced with the decision problem $(\{x, s\}, \diamond)$. This interpretation is to be contrasted with that corresponding to the statement $C(\{x, s\}, \diamond)=\{x, s\}$, in which case $s$ would also be considered choosable. Moreover, together with the absence of an alternative with the status quo property, the fact that the menu involved in this problem is a binary one makes it natural to interpret $C(\{x, s\}, \diamond)=x$ as suggestive of a strict preference of the individual for $x$ over $s$. What A2 states then is that if he thinks $s$ is strictly inferior to $x$, he continues to think that also when $s$ is the status quo.

A question that arises naturally is whether this restriction invalidates attempts to model experimental evidence suggesting that people are status quo biased. It is often the case that the bias is observed when choice is made from menus where no alternative is objectively superior to the alternative that subsequently becomes the status quo (e.g. mugs vs candy bars in Knetsch, 1989). In the case of a binary such menu, the observed behavior is explainable by letting $C(\{x, s\}, \diamond)=\{x, s\}$. The axiom allows for this choice and $C(\{x, s\}, s)=s$, a combination that indeed corresponds to status-quo biased behaviour. Seen in this way, the axiom can be thought of as ruling out potentially extremely strong status quo biases that are capable of overturning strict preference, but is absolutely permissive of such biases when the two alternatives in question are possibly indifferent or incomparable. Therefore, this axiom places an upper bound on the effect that status quo bias can have on the consumer's choices.

A comparison is in order between BSQB and the Weak Axiom of Status Quo Bias (WASQB) in Masatlioglu and Ok (2015). The latter states that, for all $x, y \in X$, $y \in C(\{x, y\}, x)$ implies $y \in C(\{x, y\}, \diamond)$ and $y \in C(\{x, y\}, \diamond)$ implies $y \in C(\{x, y\}, y)$. Clearly, the former part of BSQB implies the corresponding part of WASQB, while the second parts of the two axioms are logically distinct. Yet, it is not difficult to see that the EPD model also satisfies the second part of the WASQB axiom, and that the main model in Masatlioglu and Ok (2015) (discussed in section 4.2) is consistent with the first part of BSQB. However, the Masatlioglu-Ok model does not generally satisfy the second restriction of the BSQB axiom.

\section{A2: $a$-WARP}

For all $x, y \in X$ and $A \in \mathcal{M}$, if $C(\{x, y\}, p)=x, p \neq x$ and $y \in C(A, p)$, then $x \notin A$. 
A2 is a minimal requirement of choice consistency across decision problems with a non-specific status quo and across problems with a common specific status quo. If the statement $C(\{x, y\}, p)=x, x \neq p$, is interpreted as being suggestive of a strict preference for $x$ over $y$, as argued earlier, then the axiom's requirement that $y$ be rejected whenever $x$ is feasible and $p$ is the status quo is in line with rationality in choice, which requires that no dominated option be chosen. Obviously, the axiom's restrictions do not necessarily apply across decision problems with different status quos. The axiom is named $a$-WARP because it is a straightforward extension of the axiom under this name that is used in Gerasimou (2015). It obviously relaxes WARP by requiring no weak choice reversals between $x$ and $y$ following choice of one over the other in a $b i$ nary menu, holding the status quo fixed. As such, it is weaker than the WARP version employed in Masatlioglu and $\mathrm{Ok}$ (2015) which does impose this requirement across all problems with the same status quo.

\section{A3: Choice Implies Rejection (CIR)}

For all $(A, p) \in \mathcal{S}$, if $|A|>1$, then $C(A, p) \subset A$.

A3 requires that, irrespective of the type of decision problem he is faced with, the agent never finds himself in a situation where he might choose any of the feasible alternatives. This axiom is implied by single-valuedness of the choice correspondence which is commonly employed in the literature, but obviously does not imply it.

\section{A4: Restricted Expansion (RE)}

For all $A_{1}, \ldots, A_{k} \in \mathcal{M}$ and $x \in X$, if $x=C\left(A_{1}, \diamond\right),\left|A_{1}\right|>1$, and for $i \neq 1$ either $x \in C\left(A_{i}, \diamond\right)$ or $C\left(A_{i}^{\prime}, \diamond\right)=\emptyset$ for all $A_{i}^{\prime} \subseteq A_{i}$ with $x \in A_{i}^{\prime}$, then $x \in C\left(\bigcup_{i=1}^{k} A_{i}, \diamond\right)$.

Despite its slightly complicated statement, the behavioural restrictions of A4 are both simple and intuitive. Consider an alternative $x$ that is uniquely choosable in a decision problem with a non-specific status quo. If $x$ is also choosable (not necessarily uniquely) in each member of a given collection of decision problems of the same type, then it will also be choosable when the status quo continues to be non-specific and the problem includes all alternatives that were feasible in the individual decision problems. This restriction is essentially identical to that imposed by Weak Property $\gamma$ in Gerasimou (2015). ${ }^{4}$ However, A4 further states that even if $x$ is not choosable in any other decision problem in that collection, as long as nothing is chosen in these as well as in all smaller problems where it is feasible, $x$ will again be choosable in the larger decision problem. An intuition for the latter restriction is that if nothing is chosen over $x$ in these and in all smaller menus, then there is no evidence that $x$ is inferior to any of the alternatives in these menus. This non-inferiority, together with $x$ being uniquely best in at least one of these menus, makes it choosable in the larger decision problem.

\footnotetext{
${ }^{4}$ Property $\gamma$ is also commonly referred to as "Expansion" in the literature.
} 


\section{A5: Partial $b$-WARP}

For all $x \in X$ and $A \in \mathcal{M}$, if $x \in C(A, \diamond)$ and $|A|>1$, then $C(\{x, y\}, \diamond)=x$ for some $y \in A \backslash C(A, \diamond)$.

This axiom is a restatement (under the notation of this paper) of the axiom with the same name that was introduced in Gerasimou (2015). It states that choice of $x$ in a decision problem with a non-specific status quo implies pairwise dominance of $x$ over some $y$ that was rejected in the original problem. It is implied by WARP and hence has normative appeal. However, in a world where deferral is permissible one would also expect its descriptive relevance to increase. Intuitively, an agent whose inaction is associated with choice deferral and who escapes inaction only if he can find a suitable reason to do so, may well find such a reason in instances of pairwise dominance.

\section{Proposition 1}

The following are equivalent for a choice correspondence $C: \mathcal{S} \rightarrow X$ :

(a) $C$ satisfies $A 1-A 5$.

(b) $C$ is generated by an Extended Partial Dominance procedure.

The proof appears in Appendix A and the logical independence of the five axioms is established in Appendix B. A sketch of the proof that the axioms are sufficient for $C$ to be generated by an EPD procedure is as follows. First, the preference relation $\succ$ is defined by $x \succ y$ if $C(\{x, y\}, \diamond)$. The restricted nonempty-valuedness of $C$ in problems of the type $(A, s) \in \mathcal{S}$, together with A1 and A2 establish its acyclicity and its applicability in both types of problems. It is then shown that A2, A4 and A5 imply (1a) and A1-A3 imply (1b).

The connection between the EPD model and rational choice is explored next. Clearly, the latter kind of behavior requires that choices are always made in all decision problems of both types. Therefore, the following axiom must clearly be satisfied.

\section{A0: Decisiveness}

For all $(A, \diamond) \in \mathcal{S}, C(A, \diamond) \neq \emptyset$.

Since $C$ was taken to satisfy $C(A, s) \neq \emptyset$ by construction for all problems with a specific status quo, the above axiom adds to this restriction by ensuring that $C$ is nonempty-valued also in problems with a non-specific status quo. This obviously rules out behavioral indecisiveness and deferral.

\section{Proposition 2}

The following are equivalent for a choice correspondence $C: \mathcal{S} \rightarrow X$ : 
(a) $C$ satisfies $A 0-A 3$.

(b) There exists a unique strict linear order $\succ$ on $X$ such that, for all $(A, p) \in \mathcal{S}$

$$
C(A, p)=\{x \in A: y \nsucc x \text { for all } y \in A\} .
$$

This result clarifies that behavior which conforms with the EPD model is not inherently irrational, as it includes rational choice (with no indifference ties) as a special case whenever preferences are complete (or, equivalently, whenever the agent is forced to choose at all decision problems). The intuition is clear: When preferences are complete, the partial dominance requirement that applies to both types of decision problems becomes a requirement of total dominance, which is the hallmark of utility maximization. Here, axioms A4 and A5 are implied by the remaining ones once A0 is also assumed and are therefore redundant.

\section{Discussion}

\subsection{The Model's Predictions and the Experimental Evidence}

The EPD model explains very simply, by means of (2), the experimental evidence reported in Tversky and Shafir (1992); Luce (1998); Dhar (1997); Dhar and Simonson (2003), among others, which suggest that, when not forced to choose, people frequently defer/avoid choice in decision problems with a non-specific status quo in which they are unable to make preference comparisons. This behavior may be due to the fact that the absence of a (partially or totally) dominant option among those feasible makes choice not as easy to justify to themselves or to others (Dhar and Simonson, 2003; Simonson, 1989; Shafir, Simonson, and Tversky, 1993). Another possibility is aversion to the possible regret that may be experienced if a choice is made that later turns out to be suboptimal (Reb, 2008). It should be noted that the evidence for choice deferral mainly comes from data derived from hypothetical questionnaires, although such behavior was recently observed also in an incentivized lab experimental context in Costa-Gomes, Cueva, and Gerasimou (2014). The reported deferral rate in these studies is in the range of $20 \%-45 \%$.

On the other hand, the EPD model rules out behavior of the choice-overload type whereby decision makers defer more frequently from large menus than they do in smaller ones, possibly because they perceive them as more complex..$^{5}$ Indeed, it follows from (1a) that if choice is deferred from a large menu (and thus, in view of (2) all alternatives in that menu are mutually incomparable) but one extra partially dominant alternative is added to it, then the agent will choose that option from the expanded menu. It should be noted, however, that this prediction, although incompatible with choice overload itself, is in the same direction as to how it has been suggested that

\footnotetext{
${ }^{5}$ Evidence for this effect was first reported in Iyengar and Lepper (2000).
} 
the effect may break down once a clearly superior (totally or partially) alternative is introduced in the menu (see Scheibenhenne, Greifeneder, and Todd (2010) and references therein).

The attraction/asymmetric dominance/decoy effect, originally observed by Huber, Payne, and Puto (1982), is the phenomenon whereby introducing a dominated option to a menu of two conflict-generating alternatives increases the choice probability of the option that dominates it. The strengthening of the attraction effect when choice is not forced, defined as a significantly greater difference in the choice probabilities of the asymmetrically dominant option in the deferral vs non-deferral setting, is one of the main experimental findings in Dhar and Simonson (2003). Specifically, it was found there that although the asymmetrically dominant option tended to be uniquely chosen in the three-element menu, a significant fraction of subjects deferred at the two-element menu, thus lowering the choice probabilities of both original alternatives there. When the agent's preferences coincide with the usual partial ordering in attribute space the EPD model provides a deterministic explanation of this finding, as illustrated in the left and middle figures of Fig. 1.

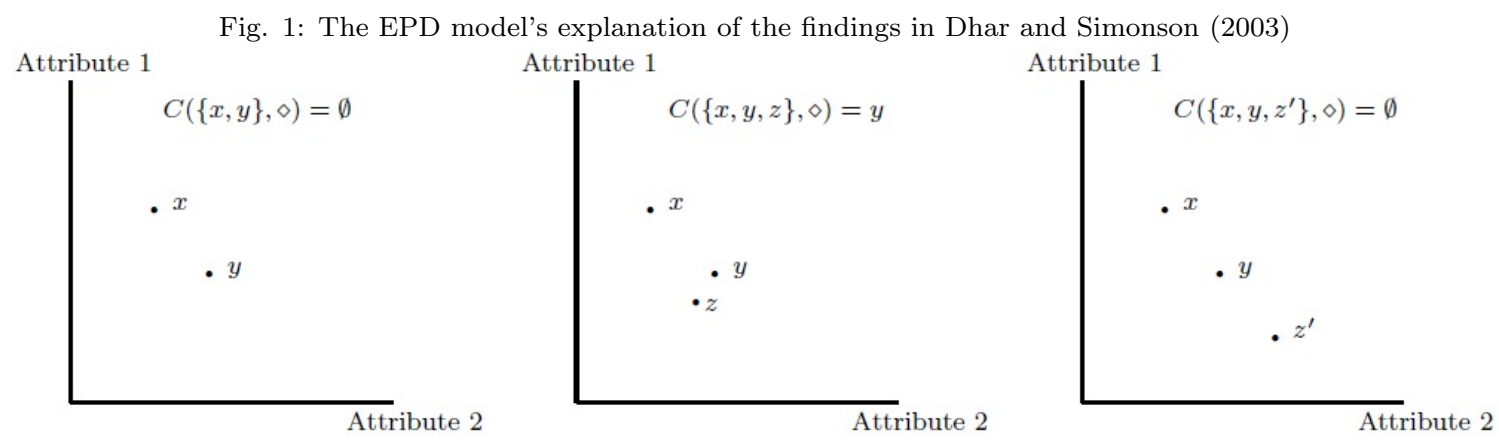

The second main finding in Dhar and Simonson (2003) is that when deferral is permissible, the magnitude of the compromise effect (defined as the increased choosability of an alternative when it is the intermediate one in a menu that also includes two extreme options) is significantly decreased. This effect occurs through the substantial deferral rates in both the two- and three-element menus when subjects are not forced to choose. The EPD model is compatible with deferring behavior in both menus when it is understood that the agent is unable to compare the three alternatives in question, as is the case for example, in the left and right figures of Fig. 1. Hence, the EPD is compatible with this finding too.

As discussed in Dhar and Simonson (2003), the relatively high incidence of deferral in the three-element menu that includes a compromise option probably reflects the reduced appeal of the latter as a reason for an agent to make a choice in a conflict situation when he is also given the opportunity to avoid choice altogether. By contrast, the robustness and strengthening of the attraction effect in this setting suggests that asymmetric dominance detection is a good-enough reason for the decision maker to choose an alternative even when deferral is possible. Both these modes of reasoning are consistent with the EPD model, which, to the author's knowledge, is the only one that 
can explain both these findings in a simple deterministic way. For a model of random choice that also accounts for these findings by introducing "perception priorities" and relaxing the Independence of Irrelevant Alternatives property on choice probabilities, however, the reader is referred to Echenique, Saito, and Tserenjigmid (2014).

When it comes to decision problems with a specific status quo, finally, the EPD model formalizes the idea that is implicit in Bewley (2002) and explicit in Mandler (2004) and Masatlioglu and Ok (2005) that status quo bias may occur because preferences are generally incomplete and the decision maker chooses to maintain the status quo option unless he can find something preferred to it, in which case he abandons it for one of these superior alternatives. In view of incompleteness, finding such a superior alternative is often impossible, hence the observed bias. In the EPD model, this bias is manifested in (3), which predicts that, as long as it is undominated, the status quo option need not be preferred to anything else feasible for it to be maintained. As already noted, an interesting feature of the EPD model is that this well-known explanation of status quo bias (which, however, has not been formalized as in (1b) and (3) before) is very symmetric to the way in which an agent defers or makes a choice in decision problems with a non-specific status quo. Specifically, in both types of problems the individual escapes the state of inaction only if the partial dominance requirement is satisfied by some undominated feasible option (in a general and in a restricted way in problems with a non-specific and a specific status quo, respectively).

\subsection{Related Literature}

The first theoretical explanation of status quo bias in a riskless setting was offered by Tversky and Kahneman (1991) (KT), who attributed its occurrence to referencedependent preferences and loss-aversion. Specifically, in one formulation of the KT model, each alternative $x$ belonged to an $n$-dimensional attribute space and, whenever this alternative was the agent's reference point, it induced a utility function $U_{x}$ such that, for every option $y, U_{x}(y)=\sum_{i=1}^{n} u_{i}\left(x_{i}-y_{i}\right)$, where $u_{i}, i \leq n$, is a strictly increasing real-valued function such that $u_{i}(0)=0, u_{i}(k)<-u_{i}(-k)$ for all $k \neq 0$. Each $u_{i}$ was assumed to be concave in the domain of attribute value gains and convex in the domain of losses. The latter two properties capture the agent's diminishing sensitivity for gains and losses, respectively, while the former ensures that the agent is loss averse in the sense that he weighs losses disproportionally more than gains of equal magnitude. For an agent who maximizes the above reference-dependent utility function and who is faced with a problem $(A, s)$ where $s$ is also the agent's reference point, this specification makes status quo bias likely whenever $A$ includes alternatives that feature sufficiently many attribute losses relative to $s$.

The main difference between the KT and the EPD model is that the latter makes a connection between problems with and without a specific status quo in such a way that in both types of problems the agent's behavior is guided by the same referenceindependent, incomplete and acyclic preference relation. Moreover, unlike the KT 
model, EPD also accounts for conflict-induced choice deferral and for the strengthening of the attraction effect in a deferral-permissive setting. On the other hand, the KT model allows for the agent's reference point to be an alternative that is still capable of influencing choice at a menu despite not being part of it. Although the EPD model features context-dependent behavior, it does not allow for non-feasible options to influence choice.

Masatlioglu and Ok (2015) (MO) model status-quo-biased choice in an abstract framework where the domain is expanded to include problems with or without an explicit (or, in our terminology, specific) status quo as in Masatlioglu and Ok (2005). In their model, the decision maker is characterized by a utility function $U$ and a correspondence $\mathcal{Q}$ on $X$ that satisfies $x \in \mathcal{Q}(x)$ for each alternative $x$. The agent's choice correspondence $C$ is such that, for all $(A, \diamond),(A, s) \in \mathcal{S}, C(A, \diamond)=\arg \max _{x \in A} U(x)$ and $C(A, s)=\arg \max _{x \in A \cap \mathcal{Q}(s)} U(x)$, respectively. Whenever he is faced with a problem of the type $(A, s)$ where $s \in A$ is his status quo, $\mathcal{Q}(s)$ is interpreted in the MO model as a psychological constraint set that contains those alternatives that are superior to $s$. Unlike the case of problems $(A, \diamond)$ with a non-specific status quo where maximization of $U$ is unconstrained and the agent is a standard utility maximizer, here $U$ is maximized over the constraint set $A \cap \mathcal{Q}(s)$. Consequently, whenever this intersection coincides with $\{s\}$, it necessarily follows that $C(A, s)=s$. However, the latter can also happen when there is more than one alternative in this intersection, and the utility function $U$ favours $s$ over those other alternatives. These are the two channels through which status quo bias can occur in this model.

The main difference between the EPD and MO models is that the latter portrays an agent who never defers and always chooses in a WARP-consistent way across problems with a common status quo. As such, it rules out deferral and asymmetric-dominance choice effects which the EPD model accounts for. Moreover, unlike the EPD procedure, the MO model assumes that the agent employs two rationales before choosing, i.e. one that is associated with $U$ and another one that is captured by $\mathcal{Q}$. Yet another difference is that, under the EPD model, the agent is never "behaviorally indifferent" between $s$ and some other alternative $y \in A$ in the sense that $y, s \in C(A, s)$. In this model, if the agent is unable to compare $y$ and $s$ by strict preference, then when it comes to choosing between these options, the tie is broken in favor of the status quo. In contrast, because the function $U$ in the MO model is not generally injective, one has $y, s \in C(A, s)$ whenever $y \in \mathcal{Q}(s)$ and $U(s)=U(y)$.

At the axiomatic level, despite the differences already mentioned in the previous section which render the two models logically distinct, the EPD model does in fact satisfy the third behavioral axiom of the MO model, called Status Quo Irrelevance. The latter states that, for any $(A, s) \in \mathcal{S}$ such that $C(B, s) \neq s$ for every $B \subseteq A$ with $|B|>1$ and $s \in B$, and such that $C(\{y, s\}, \diamond)=y$ for some $y \in A$, it holds that $C(A, s)=C(A, \diamond)$.

With regard to models of conflict-induced choice deferral, Costa-Gomes, Cueva, and 
Gerasimou (2014) proposes and axiomatically characterizes the "maximally dominant" choice rule whereby an agent who is faced with decision problems with a non-specific status quo is endowed with an incomplete but transitive weak preference relation and chooses a most preferred feasible alternative if one exists, and defers otherwise. It is shown there that such behavior is fully consistent with WARP despite incompleteness of the agent's preferences, and that it is also compatible with the choice-overload phenomenon. Compared to that model, the EPD procedure builds on the expanded choice domain, and on a strict rather than on a weak preference relation. Moreover, in addition to deferral which both models capture, EPD also explains status quo bias and asymmetric-dominance effects.

Dean (2008) also used the expanded domain and analyzed a model of choice with incomplete weak preferences in which the agent chooses a most preferred alternative if one exists. If not, he chooses the status quo when no alternative "better" than it is feasible (where "better" here is captured by a correspondence similar to $\mathcal{Q}$ in Masatlioglu and Ok (2015)), and he chooses a maximizer of the completion of his preferences otherwise (he does the same in problems with a non-specific status quo without a most preferred option). This model can account for status quo bias in large menus in the spirit of the choice overload phenomenon. However, because it builds on a nonempty-valued choice correspondence and on a completion of the agent's preferences, it is not compatible with choice deferral in the sense studied here. Finally, for a context-dependent axiomatic model of choice and deferral that is developed in a framework of preference for flexibility and regret aversion over risky objects the reader is referred to Buturak and Evren (2014).

With regard to recent models of bounded-rational choice that can explain the attraction effect, Lombardi (2009), de Clippel and Eliaz (2012), Ok, Ortoleva, and Riella (2015), Bordallo, Gennaioli, and Shleifer (2013) and Gerasimou (2015) are some recent references. These models are all reviewed in the latter paper. In the case of the first three models, the choice domain does not include decision problems with a specific (exogenous) status quo. ${ }^{6}$ Unlike the EPD model which extends the one in Gerasimou (2015) in this direction, the choice correspondence in these three models was assumed to be nonempty-valued, and it is not clear if the resulting choice procedures are robust with respect to this assumption. The model of salience-distorted, menu-dependent utility maximization in Bordallo, Gennaioli, and Shleifer (2013) is developed in the very specific domain of price-quality attribute pairs. Although the model does not allow for introducing a role for a status quo option, the possibility of deferral is captured there by the presence of the $(0,0)$-option. This inclusion, however, does not lead to the above predictions suggested in Dhar and Simonson (2003) because if the $(0,0)$-option is choosable in a menu with two price-quality conflicting products, it is also chooosable when a decoy or extreme option is also introduced.

\footnotetext{
${ }^{6}$ However, Ok, Ortoleva, and Riella (2015) do analyze a model of choice in which certain feasible alternatives act as endogenous reference points that influence behavior by restricting the set of options over which the agent is maximizing his utility function.
} 


\subsection{Remarks on the Modelling of Choice Deferral}

The possibility of allowing a choice correspondence to be empty-valued in order to model deferral that is driven by incompleteness has also been suggested in Hurwicz (1986), Clark (1995), Kreps (2012) and Costa-Gomes, Cueva, and Gerasimou (2014). Despite these works, however, there appears to be some resistance in the literature when it comes to relaxing a choice correspondence in this way. This resistance is likely due to the argument that, since not choosing is itself a choice, a specific alternative standing for "no choice" should be included in every menu and the correspondence should assign this option whenever it is understood that the agent avoids choosing.

In the author's view, when all choice objects are desirable and avoidance is exclusively due to the agent's inability to make preference comparisons, modelling deferral by means of an explicit no-choice option can be misleading. To illustrate, consider four "real" options $w, x, y$ and $z$ and let also $d$ stand for the explicit no-choice option (or non-specific status quo). Suppose the individual cannot compare $x$ and $y$ and, as a consequence, avoids choosing either of them when these are the only available options. One understands that $d$ is chosen over both $x$ and $y$ here. Suppose also that the individual prefers $x$ to $w$ and $z$, and therefore chooses $x$ from the menu that consists of these three options. In this case $x$ is chosen over $d$.

Modelled like this, the above behavior should lead one to conclude that the decision maker is irrational, since her choices violate WARP. Yet, the decision maker is simply maximizing her preferences in the second menu, and is cautiously avoiding choice in the absence of a preference comparison in the first menu. Therefore, the conclusion of irrationality here is presumably too strong. It stems from the fact that the no-choice option $d$ is treated just like every other alternative, even though when the individual actually chooses it, he doesn't do so because it is preferred to the other options, as would have been the case if avoidance had been caused by unattractiveness. Adopting the empty-set approach eliminates this problem. With it, the question of whether behavior is WARP-rational or not is answered by checking for violations of the axiom that occur only in menus where the individual does choose.

Nevertheless, introducing an explicit and always-feasible no-choice option $d$ is a reasonable modelling method in cases where avoidance is caused by the unattractiveness of all feasible alternatives. In this case the individual may well be a utility maximizer who can compare no-choice with all other options, and decide according to whether it corresponds to the highest achievable utility level or not. For instance, one may consider having no insurance to be better than having one of policies $A, B$ or $C$ and hence defer choice on the basis of this undesirability of all available policies and not because he was unable to choose among them even though all of them were thought to be better than staying uninsured. When deferral is caused exclusively by all feasible alternatives being undesirable in this sense, then both this and the empty-set modelling approach can be used to elicit the agent's preference ordering. However, in both cases this can be done only up to the desirability threshold. 
Indeed, consider first the approach where each menu $A$ becomes $A \cup\{d\}$. This implies that every binary menu $\{x, y\} \in \mathcal{M}$ becomes $\{x, y, d\} \in \mathcal{M}^{*}$, where $\mathcal{M}^{*}:=$ $\{A \cup\{d\}: A \in \mathcal{M}\}$. Let the agent's preference relation $\succsim$ on $X \cup\{d\}$ be defined by $x \succsim d$ if $x \in C(\{x, d\})$ and $x \succsim y$ if $x \in C(\{x, y, d\})$. Then, for all $w, z \in X$ such that $C(\{w, z, d\})=\{d\}$ it is impossible for the agent's preferences between $w$ and $z$ to be revealed from choices because he never chooses one of these alternatives in the presence of the other. Thus, if the agent's choices are rational, the revealed preference relation $\succsim$ will be complete and transitive on the set $Y \cup\{d\}$, where $Y=\{y \in X$ : $y \in C(\{y, d\})\}$. On the other hand, if undesirability-driven deferral is elicited via the empty-set approach, then $Y$ is defined by $Y:=\{y \in X: C(\{y\})=\{y\})$. The agent's preferences between alternatives in $Y$ will then be revealed from choices in binary menus in $\mathcal{M}$ but, again, preferences between alternatives in $X \backslash Y$ cannot be elicited.

\section{Conclusion}

The behavioral model of extended partial dominance that was presented above is the first to provide a unified explanation of choice deferral, status quo bias and the strengthening of the attraction effect/weakening of the compromise effect that have been observed when deferral is permissible. Importantly, it provides a clear theoretical distinction between the former two related but ultimately distinct phenomena. The model is the first in the literature to portray the decision maker as having a single, reference-independent, incomplete and acyclic preference relation, which is used in similar ways across decision problems of the same or of a different type. In both cases, choices are based on the principle of partial dominance. As such, it suggests the possibility that a link exists between the behavioural mechanisms underlying the attraction effect, status quo bias and avoidant behaviour more generally. Finally, the bounded irrationality of the decision rule described in the model is showcased by the fact that it actually becomes a utility-maximizing choice rule when the decision maker's preferences are complete.

\section{Appendix A: Proofs}

\section{Proof of Proposition 1:}

The proofs that the axioms are necessary and that the preference relation $\succ$ in the EPD model is unique are straightforward and omitted. For sufficiency, define $\succ$ by $x \succ y$ if $C(\{x, y\}, \diamond)=x$. Suppose $x_{1} \succ x_{2} \ldots x_{k} \succ x_{1}$ for some $x_{1}, \ldots, x_{k} \in X$. Let $A:=\left\{x_{1}, \ldots, x_{k}\right\}$. Without loss of generality, consider $\left(A, x_{1}\right) \in \mathcal{S}$. By assumption, $C\left(A, x_{1}\right) \neq \emptyset$. Suppose $x_{1} \in C\left(A, x_{1}\right)$. Then, $x_{k} \succ x_{1}$ together with A1 implies $C\left(\left\{x_{1}, x_{k}\right\}, x_{1}\right)=x_{k}$, which violates A2. Suppose instead that $x_{j} \in C\left(A, x_{1}\right)$ for some $j \neq 1$. It follows from A1 that $x_{j} \in C(A, \diamond)$. But since $x_{j-1} \succ x_{j}$ holds by assumption, this also violates $\mathrm{A} 2$. Hence, $x_{j} \notin C\left(A, x_{1}\right)$ for all $x_{j} \neq x_{1}$. It follows 
then that $C\left(A, x_{1}\right)=\emptyset$, which is impossible by assumption. Hence, $\succ$ is acyclic. Moreover, since all binary problems $(\{x, y\}, \diamond)$ are included in $\mathcal{S}, \succ$ is unique.

Consider now $(A, \diamond) \in \mathcal{S}$ and suppose $C(A, \diamond) \neq \emptyset$. Let $x \in C(A, \diamond)$. From A2, $z \nsucc x$ for all $z \in A$. From A5, $x \succ y$ for some $y \in A$. Moreover, if $x \in A$ is such that $x \succ y$ for some $y \in A$ and $z \nsucc x$ for all $z \in A$, then applying A4 as above leads to $x \in C(A, \diamond)$. It is obvious, finally, that $C(A, \diamond) \neq \emptyset$ if $C(A, \diamond)$ is as in (1a). Hence, (1a) holds.

Next, consider $(A, s) \in \mathcal{S}$. Suppose $x \in C(A, s)$ for some $x \neq s$. Assume to the contrary that there exists $y \in A$ such that $y \succ x$. It follows from $x \in C(A, s)$, $x \neq s$ and A1 that $x \in C(A, \diamond)$. But since $y \succ x$, this contradicts A2. Thus, $x \in C(A, s)$ and $x \neq s$ implies $y \nsucc x$ for all $y \in A$. Moreover, it follows from A3 that $C(\{x, s\}, s) \subset\{x, s\}$. Suppose $x=C(\{x, s\}, s)$. Then, A1 implies $x \in C(\{x, s\}, \diamond)$. From A3, $x=C(\{x, s\}, \diamond)$, or $x \succ s$. Conversely, if $C(A, s)$ consists of all $x \in A$ such that $y \nsucc x$ for all $y \in A$ and $x \succ s$, then A2 ensures that $C(A, s) \neq s$. This establishes (1b).

For completeness, we will show how the axioms also imply (2) and (3). For the former, consider $(A, \diamond) \in \mathcal{S}$ and let $x \nsucc y$ and $y \nsucc x$ for all $x, y \in A$. Assume, per contra, that $w \in C(A, \diamond)$. From A3, $C(A, \diamond) \subset A$. From A5, $C(\{w, z\}, \diamond)=w$ for some $z \in A \backslash C(A, \diamond)$. Since $w \nsucc z$ by assumption, this is a contradiction. Thus, $C(A, \diamond)=\emptyset$. Conversely, let $C(A, \diamond)=\emptyset$. Since $\succ$ is acyclic, there exists $x \in A$ such that $z \nsucc x$ for all $z \in A$. Suppose $x \succ y$ for some $y \in A$. Let $A:=\left\{x, y, z_{1}, \ldots, z_{k}\right\}$. Then, $C(\{x, y\}, \diamond)=x$ and either $x \in C\left(\left\{x, z_{i}\right\}, \diamond\right)$ or $C\left(\left\{x, z_{i}\right\}, \diamond\right)=\emptyset$ for all $i \leq k$. A4 implies $x \in C(A, \diamond)$, a contradiction. This establishes (2).

Finally, to establish (3) suppose $(A, s) \in \mathcal{S}$ is such that $y \nsucc s$ for all $y \in A$ and assume to the contrary that $x \in C(A, s)$ for some $x \neq s$. It follows from above that $x \succ s$, a contradiction. Since $C(A, s) \neq \emptyset$, this implies $s=C(A, s)$. Conversely, suppose $C(A, s)=s$ and let $x \succ s$ for some $x \in A$. A1 implies $C(\{x, s\}, s)=x$. A2 then also implies $s \notin C(A, s)$, a contradiction.

\section{Proof of Proposition 2:}

The proof that (b) implies (a) is straightforward and omitted. For the converse, define the asymmetric relation $\succ$ on $X$ as in the preceding proof. It follows from A0 and A3 that $x \succ y$ or $y \succ x$ for all $x, y \in X$. Hence, $\succ$ is complete. Suppose $x \succ y$, $y \succ z$ and $x \nsucc z$. Completeness implies $z \succ x$, i.e. $C(\{x, z\}, \diamond)=z$. By assumption, $(\{x, y, z\}, \diamond) \in Z$. Moreover, A0 implies $C(\{x, y, z\}, \diamond) \neq \emptyset$. If $C(\{x, y, z\}, \diamond)=x$, then $z \succ x$ violates A2. The cases where $C(\{x, y, z\}, \diamond)=y$ and $C(\{x, y, z\}, \diamond)=z$ are similarly ruled out. Thus, $x \succ z$ and $\succ$ is also transitive, hence a strict linear order. As above, $\succ$ is unique.

Consider $(A, \diamond) \in Z$. Suppose that $C(A, \diamond)=x$ and let $y$ be the $\succ$-maximal element of $\succ$ in $A$. Assume to the contrary that $x \neq y$. Completeness of $\succ$ implies $y \succ x$. Since $C(A, \diamond)=x$ and $y \in A$ by assumption, this violates A2. Thus, $x=y$. 
Next, let $(A, s) \in Z$ and define $x$ and $y$ as above. Consider the case where $y \neq s$ first. By way of contradiction, suppose $x \neq y$. Completeness of $\succ$ and the definition of $y$ imply $y \succ x$ and $y \succ s$. Let $x \neq s$. From A1, $x=C(A, s)$ implies $x=C(A, \diamond)$. Since $y \succ x$ and $y \in A$ by assumption, $x=C(A, \diamond)$ violates A2. Thus, $x=s$. In this case, it follows from A1 and $C(\{x, y\}, \diamond)=y$ that $C(\{s, y\}, s)=y$. Since $y \in A$ and $s=x=C(A, s)$ by assumption, this violates A2. Thus, $x=y$.

Consider finally the case where $y=s$. Suppose $x \neq y$. From the definition of $y(=s)$ one has $s \succ x$. From the definition of $x$ one also has $C(A, s)=x$. Since $x \neq s=y$, it follows from A1 and $x=C(A, s)$ that $x=C(A, \diamond)$. In view of $s \succ x$ and $s \in A$, this violates A2. Thus, $x=y$ and (1) is established for all $(A, p) \in Z$.

\section{Appendix B: Axiom Independence}

Let $X=\{w, x, y\}$ and let $\mathcal{S}$ be the union of the following collections of sets:

$$
\begin{aligned}
\{(\{w\}, \diamond),(\{x\}, \diamond),(\{y\}, \diamond),(\{w, x\}, \diamond),(\{w, y\}, \diamond),(\{x, y\}, \diamond),(\{w, x, y\}, \diamond)\}, \\
\{(\{w\}, w),(\{w, x\}, w),(\{w, y\}, w),(\{w, x, y\}, w)\}, \\
\\
\{(\{x\}, x),(\{w, x\}, x),(\{x, y\}, x),(\{w, x, y\}, x)\}, \\
\\
\{(\{y\}, y),(\{w, y\}, y),(\{x, y\}, y),(\{w, x, y\}, y)\}
\end{aligned}
$$

Each of the examples below presents a set of choices that conforms with all but one of the axioms of Proposition 1. Axiom A1 is broken into A1a and A1b to reflect the former and the latter condition in the statement of the axiom, respectively.

\section{Not A1a}

$$
\begin{gathered}
C(\{w, x\}, \diamond)=C(\{x, y\}, \diamond)=C(\{w, x, y\}, \diamond)=x, \quad C(\{w, y\}, \diamond)=\emptyset \\
C(\{w, x\}, w)=C(\{w, x, y\}, w)=x, \quad C(\{w, y\}, w)=w \\
C(\{w, x\}, x)=C(\{w, x, y\}, x)=w, \quad C(\{x, y\}, x)=x \\
C(\{w, y\}, y)=C(\{w, x, y\}, y)=y, \quad C(\{x, y\}, y)=x
\end{gathered}
$$

Not A1b

$$
\begin{gathered}
C(\{w, x\}, \diamond)=\emptyset, \quad C(\{w, y\}, \diamond)=C(\{w, x, y\}, \diamond)=w, \quad C(\{x, y\}, \diamond)=x \\
C(\{w, x\}, w)=C(\{w, y\}, w)=C(\{w, x, y\}, w)=w \\
C(\{w, x\}, x)=C(\{w, x, y\}, x)=w, \quad C(\{x, y\}, x)=x
\end{gathered}
$$




$$
C(\{w, y\}, y)=C(\{x, y\}, y)=C(\{w, x, y\}, y)=y
$$

Not A2

$$
\begin{gathered}
C(\{w, x\}, \diamond)=C(\{w, x, y\}, \diamond)=w, \quad C(\{w, y\}, \diamond)=C(\{x, y\}, \diamond)=y \\
C(\{w, x\}, w)=C(\{w, x, y\}, w)=w, \quad C(\{w, y\}, w)=y \\
C(\{w, x, y\}, x)=x, \quad C(\{w, x\}, x)=w, \quad C(\{x, y\}, x)=y \\
C(\{w, y\}, y)=C(\{x, y\}, y)=C(\{w, x, y\}, y)=y .
\end{gathered}
$$

Not A3

$$
\begin{gathered}
C(\{w, x\}, \diamond)=\{w, x\}, \quad C(\{w, y\}, \diamond)=w, \quad C(\{x, y\}, \diamond)=x, \quad C(\{w, x, y\}, \diamond)=\{w, x\}, \\
C(\{w, x\}, w)=C(\{w, y\}, w)=C(\{w, x, y\}, w)=w \\
C(\{w, x\}, x)=\{w, x\}, \quad C(\{x, y\}, x)=x, \quad C(\{w, x, y\}, x)=w \\
C(\{w, y\}, y)=C(\{w, x, y\}, y)=w, \quad C(\{x, y\}, y)=x
\end{gathered}
$$

Not A4

$$
\begin{gathered}
C(\{w, x\}, \diamond)=C(\{w, y\}, \diamond)=\emptyset, \quad C(\{x, y\}, \diamond)=x, \quad C(\{w, x, y\}, \diamond)=\emptyset, \\
C(\{w, x\}, w)=C(\{w, x, y\}, w)=C(\{w, y\}, w)=w \\
C(\{w, x\}, x)=C(\{w, x, y\}, x)=C(\{x, y\}, x)=x \\
C(\{w, y\}, y)=C(\{w, x, y\}, y)=y, \quad C(\{x, y\}, y)=x
\end{gathered}
$$

Not A5

$$
\begin{gathered}
C(\{w, x\}, \diamond)=C(\{x, y\}, \diamond)=\emptyset, \quad C(\{w, x, y\}, \diamond)=\{w, x\}, \quad C(\{w, y\}, \diamond)=w \\
C(\{w, x\}, w)=C(\{w, x, y\}, w)=C(\{w, y\}, w)=w \\
C(\{w, x\}, x)=C(\{w, x, y\}, x)=C(\{x, y\}, x)=x \\
C(\{w, y\}, y)=C(\{w, x, y\}, y)=w, \quad C(\{x, y\}, y)=y
\end{gathered}
$$

\section{References}

Anderson, C. J. (2003): "The Psychology of Doing Nothing: Forms of Decision Avoidance Result from Reason and Emotion," Psychological Bulletin, 129, 139-167.

Apesteguia, J., and M. Ballester (2009): "A Theory of Reference-Dependent Behavior," Economic Theory, 40, 427-455.

Bewley, T. F. (2002): "Knightian Decision Theory. Part I," Decisions in Economics and Finance, 25, 79-110. 
Bleichrodt, H. (2007): "Reference-Dependent Utility with Shifting Reference Points and Incomplete Preferences," Journal of Mathematical Psychology, 51, 266276.

Bordallo, P., N. Gennaioli, and A. Shleifer (2013): "Salience and Consumer Choice," Journal of Political Economy, 121, 803-843.

Buturak, G., and O. Evren (2014): "A Theory of Choice When "No Choice" is an Option," mimeo.

Clark, S. A. (1995): "Indecisive Choice Theory," Mathematical Social Sciences, 30, $155-170$.

Costa-Gomes, M., C. Cueva, and G. Gerasimou (2014): "Choice, Deferral and Consistency," Discussion Paper 1416, University of St Andrews.

de Clippel, G., and K. Eliaz (2012): "Reason-Based Choice: A Bargaining Rationale for the Attraction and Compromise Effects," Theoretical Economics, 7, 125-162.

Dean, M. (2008): "Status Quo Bias in Large and Small Choice Sets," mimeo.

Dhar, R. (1997): "Consumer Preference for a No-Choice Option," Journal of Consumer Research, 24, 215-231.

Dhar, R., and I. Simonson (2003): "The Effect of Forced Choice on Choice," Journal of Marketing Research, 40, 146-160.

Echenique, F., K. Saito, and G. Tserenjigmid (2014): "The PerceptionAdjusted Luce Model," mimeo.

Gerasimou, G. (2015): "Partially Dominant Choice," Economic Theory, forthcoming.

Greenleaf, E. A., and D. R. Lehmann (1995): "Reasons for Substantial Delay in Consumer Decision Making," Journal of Consumer Research, 22, 186-199.

Huber, J., J. W. Payne, and C. Puto (1982): "Adding Asymmetrically Dominated Alternatives: Violations of Regularity and the Similarity Hypothesis," Journal of Consumer Research, 9, 90-98.

Hurwicz, L. (1986): "On the Implementation of Social Choice Rules in Irrational Societies," in Essays in Honor of Kenneth J. Arrow, ed. by W. P. Heller, R. M. Starr, and D. A. Starett, vol. I, chap. 4. Cambridge University Press.

Iyengar, S. S., and M. R. Lepper (2000): "When Choice is Demotivating: Can One Desire Too Much of a Good Thing?," Journal of Personality and Social Psychology, 79, 995-1006.

Knetsch, J. L. (1989): "The Endowment Effect and Evidence of Nonreversible Indifference Curves," American Economic Review, 79, 1277-1284. 
Knetsch, J. L., and J. A. Sinden (1984): "Willingness to Pay and Compensation Demanded: Experimental Evidence of an Unexpected Disparity in Measures of Value," Quarterly Journal of Economics, 99, 507-521.

Kreps, D. M. (2012): Microeconomic Foundations I: Choice and Competitive Markets. Princeton NJ: Princeton University Press.

Kriesler, K., and S. Nitzan (2008): "Is Context-Based Choice Due to ContextDependent Preferences," Theory and Decision, 64, 65-80.

Lombardi, M. (2009): "Reason-Based Choice Correspondences," Mathematical Social Sciences, 57, 58-66.

Luce, M. F. (1998): "Choosing to Avoid: Coping with Negatively Emotion-Laden Consumer Decisions," Journal of Consumer Research, 24, 409-433.

Mandler, M. (2004): "Status Quo Maintenance Reconsidered: Changing or Incomplete Preferences?," Economic Journal, 114, F518-F535.

Masatlioglu, Y., and E. A. Ok (2005): "Rational Choice with Status Quo Bias," Journal of Economic Theory, 121, 1-29.

(2015): "A Canonical Model of Choice with Initial Endowments," Review of Economic Studies, forthcoming.

OK, E. A., P. Ortoleva, and G. Riella (2015): "Revealed (P)reference Theory," American Economic Review, forthcoming.

ReB, J. (2008): "Regret Aversion and Decision Process Quality: Effects of Regret Salience on Decision Process Carefulness," Organizational Behavior and Human Decision Processes, 105, 169-182.

Samuelson, W., and R. Zeckhauser (1988): "Status Quo Bias in Decision Making," Journal of Risk and Uncertainty, 1, 7-59.

Scheibenhenne, B., R. Greifeneder, and P. M. Todd (2010): "Can There Ever Be Too Many Options? A Meta-Analytic Review of Choice Overload," Journal of Consumer Research, 37, 409-425.

Shafir, E., I. Simonson, and A. Tversky (1993): "Reason-Based Choice," Cognition, 11, 11-36.

Simonson, I. (1989): "Choice Based on Reasons: The Case of Attraction and Compromise Effects," Journal of Consumer Research, 16, 158-174.

Tversky, A., and D. Kahneman (1991): "Loss Aversion in Riskless Choice: A Reference-Dependent Model," Quarterly Journal of Economics, 106, 1039-1061.

Tversky, A., and E. Shafir (1992): "Choice under Conflict: The Dynamics of Deferred Decision," Psychological Science, 3, 358-361.

Zakay, D. (1984): "To Choose or not to Choose": On Choice Strategy in Face of a Single Alternative," American Journal of Psychology, 97, 373-389. 